Reprod. Nutr. Dévelop., 1988, 28 (1), 87-88.

\title{
Courbes d'excrétion fécale de l'oxyde de chrome administré aux vaches laitières au pâturage. Définition d'un échantillonnage biquotidien des fèces
}

\author{
Nicole BARTIAUX-THILL, R. OGER, E. FRANÇOIS, A. THEWIS $\left(^{*}\right)$ \\ Centre de Recherches Agronomiques de l'Etat, \\ Avenue de la Faculté d'Agronomie, 22, B-5800 Gembloux, Belgique. \\ (*) Faculté des Sciences Agronomiques de l'Etat, \\ Chaire de Zootechnie, Passage des Déportés, 2, B-5800 Gembloux, Belgique.
}

Summary. Making allowance for a periodicity of $\mathrm{Cr}_{2} \mathrm{O}_{3}$ concentration in the faeces during a 24-hour period, we developed a faecal sampling procedure, simple and compatible with field conditions.

La précision de l'estimation de la concentration journalière moyenne en $\mathrm{Cr}_{2} \mathrm{O}_{3}$ lors d'un échantillonnage biquotidien des fèces, dépend de l'amplitude et de la répétabilité des cycles d'excrétion du $\mathrm{Cr}_{2} \mathrm{O}_{3}$ (Langlands, 1975). Le présent travail a pour objectif de définir un échantillonnage biquotidien des fèces à partir des courbes d'évolution fécales du $\mathrm{Cr}_{2} \mathrm{O}_{3}$ obtenues dans différentes conditions expérimentales.

Matériel et méthodes. Dans deux essais, au pâturage (prairie permanente à prédominance de ray-grass anglais), sur 6 lots de 3 vaches en lactation nous avons établi 72 courbes journalières d'excrétion fécale du $\mathrm{Cr}_{2} \mathrm{O}_{3}$. Ces lots se différencient par l'apport de concentrés (c : faible - $C$ : élevé) et (ou) la quantité $\mathrm{d}^{\prime}$ herbe offerte/animal/jour (h : faible - $\mathrm{H}$ : élevé, tabl. 1).

TABL. 1. - Conditions expérimentales.

\begin{tabular}{|c|c|c|c|c|c|c|}
\hline \multirow{2}{*}{$\begin{array}{l}N^{\circ} \text { de l'essai } \\
N^{\circ} \text { du lot }\end{array}$} & \multicolumn{2}{|c|}{1} & \multicolumn{4}{|c|}{ II } \\
\hline & $A(h . c)$ & $B($ h.C) & $\mathrm{C}(\mathrm{H} . \mathrm{C})$ & $D\left(H_{.} . c\right)$ & $E$ (h.C) & $F(h . c)$ \\
\hline Herbe offerte* & 12,6 & 12,6 & 22,7 & 22,7 & 12,2 & 12,2 \\
\hline Digestibilité $M S$ ration ( $M \%$ ) & 75,8 & 77,8 & 76,5 & 76,2 & 74,0 & 73,4 \\
\hline Concentrés* & 1,7 & 6,2 & 6,3 & 2,7 & 6,3 & 2,7 \\
\hline Ingestion totale ${ }^{*}$ & 12,4 & 19,7 & 20,5 & 17,8 & 18,6 & 15,0 \\
\hline Ingestion herbe ${ }^{*}$ & 10,7 & 13,5 & 14,2 & 15,1 & 12,3 & 12,2 \\
\hline Production lait** & 16,3 & 31,4 & 29,0 & 20,2 & 29,0 & 20,2 \\
\hline
\end{tabular}

* en $\mathrm{kg} \mathrm{MS/animal/jour} \mathrm{;} \mathrm{**} \mathrm{en} \mathrm{kg} \mathrm{lait} \mathrm{à} 4 \% \mathrm{MG}$.

L'oxyde de chrome, en poudre, est administré, en 2 fois, aux heures de traite : $7 \mathrm{~h}$ et $17 \mathrm{~h}$, à raison de $20 \mathrm{~g} / \mathrm{jour} /$ animal inclus dans $200 \mathrm{~g}$ d'agglomérés de luzerne. L'échantillonnage des fèces s'effectue au pâturage, pendant 4 jours consécutifs, sur toutes les bouses, toutes les heures entre $6 \mathrm{~h}$ et $22 \mathrm{~h}$; les défécations de la nuit sont échantillonnées à $6 \mathrm{~h}$; dès lors, les données relatives aux observations effectuées à $6 \mathrm{~h}$ représentent la concentration moyenne des fèces émises entre $22 \mathrm{~h}$ et $6 \mathrm{~h}$. Le marqueur est dosé selon la méthode décrite par François et al. (1978). 
Résultats et discussion. Etant donné que tous les animaux ne défèquent pas nécessairement pendant chaque intervalle de temps, les résultats qui sont présentés à la figure 1 sont en réalité des valeurs estimées issues d'une analyse de la variance non orthogonale avec comme facteurs principaux les animaux et les jours. Les courbes d'excrétion fécale, relatives aux divers lots, ne sont pas parallèles ce qui montre que l'amplitude des variations de la concentration en $\mathrm{Cr}_{2} \mathrm{O}_{3}$ est différente suivant les lots. Si l'on compare des lots avec $\mathrm{c}$ ou avec $\mathrm{C}$, les concentrations en $\mathrm{Cr}_{2} \mathrm{O}_{3}$ sont toujours inférieures pour les lots $\mathrm{C}$ car l'ingestion totale est plus élevée pour ces lots. Entre les lots $A$ (h.c) et $B$ (h.C) l'écart est plus accentué car à une forte différence d'ingestion, s'ajoute une différence dans les digestibilités. Cependant, il existe une certaine périodicité dans l'évolution de la concentration fécale en $\mathrm{CR}_{2} \mathrm{O}_{3}$ au cours du nycthémère.

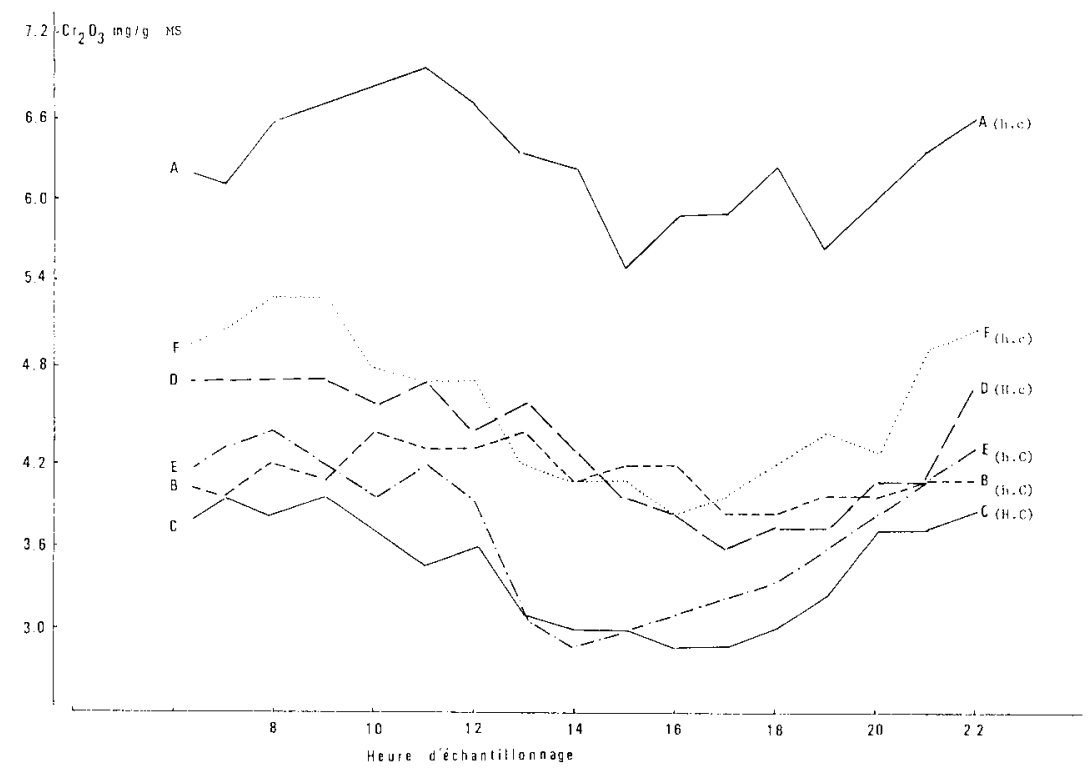

FIG. 1. - Evolution journalière de la concentration fécale en $\mathrm{Cr}_{2} \mathrm{O}_{3}$. Quantité d'herbe offerte: h (faible), H (élevée); Niveau de concentré : c (faible), C (élevé). A : h.C ; B : h.C ; C : H.C ; D : H.c ; E : h.C ; F : h.c.

En effet, les teneurs en $\mathrm{Cr}_{2} \mathrm{O}_{3}$ augmentent dans les heures qui suivent l'administration du marqueur, notamment après $17 \mathrm{~h}$. Par contre, des teneurs plus faibles sont mesurées entre $13 \mathrm{~h}$ et $17 \mathrm{~h}$. La périodicité a été mise à profit pour dégager un schéma d'échantillonnage de fèces, après traitement statistique des données, à partir de l'écart $\left(\bar{x}_{24}-\bar{x}_{b}\right)$ entre la moyenne $\left(\bar{x}_{24}\right)$ obtenue pour l'ensemble des observations pendant $24 \mathrm{~h}$ et la moyenne $\left(\bar{x}_{b}\right)$ d'un échantillonnage biquotidien, ainsi que de l'écart type ${ }^{(s)}$ des écarts entre $\bar{x}_{24}$ et $\bar{x}_{b}$. La détermination de la concentration journalière moyenne, indépendamment de la disponibilité en herbe et du niveau de complémentation, est très précise si l'on effectue un prélèvement à $8 \mathrm{~h}$ et un autre à $16 \mathrm{~h}\left(\bar{x}_{24} \bar{x}_{\mathrm{b}}=0,01, \mathrm{~s}=0,05\right)$.

François E., Thill N., Théwis A., 1978. Ann. Zootechn., 27, 355-361.

Langlands J. P., 1975. In Digestion and metabolism in the ruminants. Ed. by McDonald I. W. et Warner A. C. I., 320-332. 